\title{
Sensitive Diagnostics for Chemically Reacting Flows
}

\author{
Aamir Farooq* \\ *Clean Combustion Research Center, Division of Physical Sciences and Engineering, King Abdullah University of \\ Science and Technology (KAUST), Thuwal, Saudi Arabia \\ aamir.farooq@kaust.edu.sa
}

Increasingly stringent emission regulations, global warming, and depleting fossil fuel resources are driving the design of combustion systems towards extreme operating conditions. Ultra-lean fuel mixtures, very high pressures, and low temperature combustion are some of the directions being pursued currently. In such domains, fuel chemistry and reaction kinetics play critical role in the design and optimization of combustion systems.

Common facilities for the study of homogeneous chemical kinetics include shock tubes, rapid compression machines, combustion bombs, flow reactors, well-stirred reactors, and motored engines. Of these, shock tubes provide the most ideal environment to investigate fuel chemistry in the absence of heat transfer and fluid dynamic effects. Shock tubes are generally used to measure ignition delay times of fuel/oxidizer mixtures under varying conditions of temperature, pressure, and stoichiometry. However, when coupled with in-situ sensitive diagnostics, shock tubes can provide measurements of reaction rate coefficients and species time-history profiles. Such data are extremely useful in the development and validation of detailed chemical kinetic models.

This talk will feature latest diagnostic developments for sensitive detection of gas temperature [1] and important combustion species, such as ethylene [2], acetylene [3], propene [4], methane [5], and hydrogen peroxide [6]. Advanced optical strategies, such as intrapulse chirping, wavelength modulation, and cavity ringdown are employed. It will be demonstrated how the applications of these diagnostics enable deeper insights into complex reaction mechanisms and chemically reacting flows.

\section{References:}

[1] R.S.M. Chrystie, E.F. Nasir, A. Farooq, "Ultra-fast and calibration-free temperature sensing in the intrapulse mode", Optics Letters 39 6620-6623 (2014)

[2] A. Alquaity, E. Essebbar, A. Farooq, "Sensitive and ultra-fast detection using pulsed cavity ringdown spectroscopy", Optics Express 23 7217-7226 (2015)

[3] U. KC, E.F. Nasir, A. Farooq, "A mid-infrared absorption diagnostic for acetylene detection", Applied Physics B (DOI: 10.1007/s00340-015-6125) (2015)

[4] R.S.M. Chrystie, E.F. Nasir, A. Farooq, "Propene concentration sensing for combustion gases using quantum-cascade laser absorption near $11 \mu \mathrm{m}$ ", Applied Physics B (DOI: 10.1007/s00340-015-6139-4) (2015)

[5] M.B. Sajid, T. Javed, A. Farooq, "High-temperature measurements of methane and acetylene using quantum cascade laser absorption near $8 \mu \mathrm{m}$ ", Journal of Quantitative Spectroscopy and Radiative Transfer 155 66-74 (2015)

[6] M.B. Sajid, E. Essebbar, T. Javed, C. Fittschen, A. Farooq, "Measurement of the rate of hydrogen peroxide thermal decomposition in a shock tube using quantum cascade laser absorption near $7.7 \mu \mathrm{m}$ ", International Journal of Chemical Kinetics 46275 - 284 (2014)

OCIS Codes: (120.1740) Combustion diagnostics; (280.1545) Chemical analysis; (300.1030) Absorption. 